\title{
LOWER BOUNDS FOR THE L.-S. CATEGORY OF PRODUCTS
}

\author{
BARRY JESSUP AND BITJONG NDOMBOL
}

(Communicated by Frederick R. Cohen)

\begin{abstract}
Halperin and Lemaire introduced L.-S. category type invariants left$\operatorname{Mcat}(A)$ and $\operatorname{right}-\operatorname{Mcat}(A)$ for certain differential algebras $(A, d)$. In particular, they proved that if $(A, d)=C^{*}(S, k)$ is the $k$-valued singular cochains on 1-connected space $S$, then these invariants are lower bounds for the classical category cat $(S)$. We use an explicit model for Ganea's space due to Felix, Halperin, Lemaire, and Thomas to prove $1 \mathrm{Mcat}(A \otimes B) \leq 1 \mathrm{Mcat}(A)+e(B)$, over any field, where $e$ denotes Toomer's invariant. This proves Ganea's conjecture for Mcat over fields of arbitrary characteristic.
\end{abstract}

\section{INTRODUCTION}

Recently, Halperin and Lemaire introduced a hierarchy of homotopy invariants (lMcat, rMcat, Acat) for certain differential graded algebras over a field $k$ and examples of Idrissi [I] have shown that these are all distinct. For the case of singular $k$-valued cochains on a simply connected space $S$, they are all lower bounds for the Lusternik-Schnirelmann category, cat $(S)$, and can depend on the chosen field, though only on its characteristic [N].

The classical category (which is the least number less one of open subsets, contractible in $S$, needed to cover $S$ ) is usually difficult to compute, and lower bounds such as Toomer's invariant, $e_{k}(S)$, are particularly interesting and useful. It is known, for example, that $e_{k}$ is additive on products [T]. Some efforts to find lower bounds for the aforementioned approximations to cat $(S)$, particularly on product spaces, have met with some limited success [J, N], and these helped in the proof of Ganea's conjecture for rational spaces $[\mathrm{H}]$. Here we use similar techniques to prove

Theorem. If $(A, d)$ and $(B, d)$ are differential graded cochain algebras over $k$ with 1-connected cohomology of finite type, then

$$
\operatorname{IMcat}_{k}(A)+\operatorname{IMcat}_{k}(B) \geq \operatorname{IMcat}_{k}(A \otimes B) \geq \operatorname{IMcat}_{k}(A)+e_{k}(B)
$$

and an identical inequality is also satisfied by $\mathrm{rMcat}_{k}$.

For the special case $(A, d)=C^{*}(S, k)$ we prove

Received by the editors March 15, 1990 and, in revised form, July 25, 1990.

1991 Mathematics Subject Classification. Primary 55M30, 55P60, 55P62.

Key words and phrases. Lusternik-Schnirelmann category, free tensor model, Ganea's conjecture. The first author's research was partially supported by an NSERC operating grant. 
Corollary 1. If $S$ and $T$ are simply connected spaces with the homotopy type of $C W$-complexes and $H^{*}(S, k)$ and $H^{*}(T, k)$ are of finite $k$-type, then

$$
\operatorname{Mcat}_{k}(S)+\operatorname{Mcat}_{k}(T) \geq \operatorname{Mcat}_{k}(S \times T) \geq \operatorname{Mcat}_{k}(S)+e_{k}(T) .
$$

In particular,

Corollary 2. Ganea's conjecture is true for Mcat $_{k}$, i.e., if $T$ is a simply connected finite $C W$-complex, then

$$
\operatorname{Mcat}_{k}\left(T \times S^{n}\right)=\operatorname{Mcat}_{k}(T)+1 .
$$

We remark that $e_{k}$ and Mcat $_{k}$ depend only on the characteristic of $k[\mathrm{~N}]$.

\section{NOTATION AND KNOWN RESULTS}

We will use the notation and conventions of [HL] throughout. All algebras, vector spaces, and their tensor products are over a field $k$ of arbitrary characteristic. Any DGA $(C, d)$ (differential graded algebra) whose homology has finite type and is 1 -connected has a model $(A, d)$ in $k$ - $\mathrm{DGA}_{1}^{*}$, the cofibration category of connected, 1 -connected associative differential graded cochain algebras of finite type. That is, there is a map $\varphi:(A, d) \cong(C, d)$, which induces an isomorphism on cohomology (hereafter, such a map will be called a quism). There is a notion of homotopy in $k$ - $\mathrm{DGA}_{1}^{*}$, and the invariants we describe are invariants of homotopy type; in particular, they are preserved by quisms.

Any $(A, d)$ in $k$ - $\mathrm{DGA}_{1}^{*}$ has a free tensor model, i.e., there is a quism of DGAs $(T(V), d) \stackrel{\cong}{\rightrightarrows}(A, d)$ where $T(V)$ is the tensor algebra on a graded vector space $V$ over $k$ of finite type: $V=\bigoplus V^{j}, j \geq 2$. We may also suppose that the model is minimal, i.e., the differential $d: V \rightarrow T^{\geq 2}(V)$, where as usual $T^{p}(V)=V^{\otimes p}=V \otimes \cdots \otimes V$ ( $p$ times). Morphisms in $k$ - $\mathrm{DGA}_{1}^{*}$ have free minimal models also. We can factor $A \stackrel{\varphi}{\rightarrow} B$ as $A \stackrel{i}{\rightarrow} A \amalg T(V) \stackrel{\cong}{\rightarrow} B$, where $A \stackrel{i}{\rightarrow} A \amalg T(V)$ is a minimal free extension and $\simeq$ denotes a quism.

We now define the homotopy invariants $\mathrm{IMcat}_{k}, \mathrm{rMcat}_{k}$, Acat , and $_{k}$. Let $(T(V), d) \rightarrow(A, d)$ be a free minimal model for $(A, d)$, and suppose that $(T(V), d) \stackrel{j}{\rightarrow}\left(T\left(V \oplus W_{m}\right), d\right) \cong\left(T(V) / T^{>m}(V), \bar{d}\right)$ is a free model for the projection $T(V) \stackrel{\pi}{\rightarrow} T(V) / T^{>m}(V)$. We call $T(V) / T^{m}(V)$ the $m$ th Ganea space of $A$. Call set maps $r:\left(T\left(V \oplus W_{m}\right), d\right) \rightarrow(T(V), d)$, which commute with the differentials and which satisfy $r \circ j=\mathrm{id}_{T(V)}$ retracts. Then

(1) $\operatorname{Acat}_{k}(A)$ is the least $m$ for which there is a retract, which is a morphism of graded algebras.

(2) $\operatorname{lMcat}_{k}(A)\left(\operatorname{resp} . \operatorname{rMcat}_{k}(A)\right)$ is the least $m$ for which there is a retract, which is a morphism of graded left- $T(V)$ modules (resp. right- $T(V)$ modules).

(3) $e_{k}(A)$ is the least $m$ for which there is a retract, which is a morphism of linear spaces.

Note that Toomers's invariant $e_{k}(A)$ is also the largest $m$ for which some nontrivial class in $H^{*}(T(V)) \cong H^{*}(A)$ is represented by a cycle in $T^{\geq m}(V)$. Clearly, $e_{k}(A) \leq \min \left\{\operatorname{lMcat}_{k}(A), \operatorname{rMcat}_{k}(A)\right\} \leq \max \left\{\operatorname{lMcat}_{k}(A), \operatorname{rMcat}_{k}(A)\right\}$ $\leq \operatorname{Acat}_{k}(A)$.

In the case when $A=C^{*}(S)$, Halperin and Lemaire observed that $A \cong A^{\text {opp }}$ and so $\operatorname{IMcat}_{k}(A)=\operatorname{rMcat}_{k}(A) \equiv \operatorname{Mcat}_{k}(S)$. If $S$ is simply connected, they 
also proved that $\operatorname{cat}(S) \geq \operatorname{Acat}_{k}(S)$, showing that $\operatorname{Mcat}_{k}(S)$ is also a lower bound for the classical category of a space.

The idea of our proof of the theorem stated above is similar to that in $[\mathrm{J}]$ but we can be explicit because of an efficient model for $\left(T(V) / T^{>m}(V), \bar{d}\right)$ given in [FHLT]. We describe that model here only for use in computations for IMcat ; mirror image constructions yield the required models for rMcat. (See, e.g., $[\mathrm{N}, \mathrm{I}]$.

Recall that $T(V)$ has a bigrading $T(V)^{p, q}=\left[T^{p}(V)\right]^{p+q}$ where $p+q$ is the total (topological) degree. This bigrading of $T(V)$ above can be carried to $T(V) / T^{>m}(V)$ by making $\pi$ homogeneous of bidegree $(0,0)$. Let $M=$ $M^{m, *}=s\left(T^{m+1}(V)\right)$, i.e., $M^{m, q}=s[T(V)]^{m+1, q}$. There is an isomorphism $\omega: T(V) \otimes M \stackrel{\cong}{\rightarrow} T^{>m}(V)$ defined by $\omega(\Phi \otimes s \psi)=(-1)^{\mid \Phi} \mid \Phi \cdot \psi$ where $|\Phi|$ is the topological degree of $\Phi$. We can then define a differential $D$ on $T(V) \otimes(k \oplus M)$ by $D=d$ on $T(V) \otimes k$ and $D=\omega-\omega^{-1} d \omega$ on $T(V) \otimes M$. This makes $(T(V) \otimes(k \oplus M), D)$ into a differential left $T(V)$-module and an easy calculation shows that the map $(T(V) \otimes(k \oplus M), D) \stackrel{\psi}{\rightarrow}\left(T(V) / T^{>m}(V), \bar{d}\right)$, defined by $\psi(v)=\pi(v)$ and $\psi(T(V) \otimes M)=0$, is a quism. Using [HL, Proposition A1(i)], we can lift the quism $\left(T\left(V \oplus W_{m}\right), d\right) \stackrel{\simeq}{\rightrightarrows}\left(T(V) / T^{>m}(V), \bar{d}\right)$ over $\Psi$ to a quism $\left(T\left(V \oplus W_{m}\right), \bar{d}\right) \rightarrow(T(V) \otimes(k \oplus M), D)$. Thus any retract of our new model is one of the free model and we can use our new model to compute lMcat .

Let $(T(U), d)$ and $(T(V), d)$ be minimal free tensor models for $A$ and $B$ respectively, and let $(T(U \oplus V), d)=(T(U), d) \amalg(T(V), d)$ be the coproduct in the category of DGAs. We construct a free model for the tensor product as follows. (See Ndombol [N] for an alternate construction, which provides an explicit formula for the differential.) Define a DGA morphism $\varphi^{\prime}$ : $(T(U \oplus V), d) \rightarrow(T(U), d) \otimes(T(V), d)$ by $\varphi^{\prime} u=u \otimes 1$ and $\varphi^{\prime}(v)=1 \otimes v$. Thus $\varphi$ preserves the obvious inclusions of $T(U)$ and $T(V)$. Now let

$$
(T(U \oplus V), d) \rightarrow(T(U \oplus V \oplus W), d) \stackrel{\varphi}{\rightarrow}((T(U), d) \otimes(T(V), d))
$$

be a minimal free model for $\varphi^{\prime}$. We can thus use $(T(U \oplus V \oplus W), d)$ to compute 1 Mcat $(A \otimes B)$, since if $T(U) \stackrel{\varphi_{A}}{\rightarrow} A$ and $T(V) \stackrel{\varphi_{B}}{\longrightarrow} B$ are minimal free models for $A$ and $B$ in $\mathrm{ADG}_{1}^{*}$, then $\left(\varphi_{A} \otimes \varphi_{B}\right) \circ \varphi$ exhibits $(T(U \oplus V \oplus W), d)$ as a minimal free tensor model for $(A \otimes B, d)$.

We remark that Corollary 1 follows from the theorem and the fact that $C^{*}(S \times T) \sim C^{*}(S) \otimes C^{*}(T)$.

\section{Proof of THE THEOREM}

The first inequality of the theorem is proven in a manner similar to that for Mcat or cat $[\mathrm{FH}, \mathrm{J}]$. We refer the reader to [N] for details. We show here that $\operatorname{lMcat}(A \otimes B)-e(B) \geq \operatorname{lMcat}(A)$. The argument is essentially the second oldest in the book. Beginning in the $m$ th Ganea space of $A$, we "multiply" by a longest representative $\alpha$ of a cohomology class in $B$ (this is the map $\beta$ ) and then use the retract guaranteed by 1 Mcat $(A \otimes B) \leq m+n$ and $\varphi$ to get back to $A \otimes B$. Since $[\alpha] \neq 0$, we can "divide" by $\alpha$ and return to $A$. The composition of these maps is the required retract.

So assume that $e(B)=n$ and $1 \mathrm{Mcat}(A \otimes B)=m+n$. (Since $1 \mathrm{Mcat}(A \otimes B) \geq$ $e(A \otimes B)=e(A)+e(B), m \geq 0$.) Let $X, M$, and $N$ denote $U \oplus V \oplus W$, 
$s T^{m+1}(U)$, and $s T^{m+n+1}(X)$ respectively, and let

$$
\begin{aligned}
& \left(T(U) \otimes(k \oplus M), D_{U}\right) \rightarrow\left(T(U) / T^{>m}(U), \bar{d}\right), \\
& \left(T(X) \otimes(k \oplus N), D_{X}\right) \rightarrow\left(T(X) / T^{>m+n}(X), \bar{d}\right)
\end{aligned}
$$

be the models for the projections as described in $\S 2$. Let

$$
R:\left(T(X) \otimes(k \oplus N), D_{X}\right) \rightarrow\left(T(X), d_{X}\right)
$$

be the left- $T(X)$ module map guaranteed by $\mathrm{lMcat}(A \otimes B)=m+n$, and assume that $\alpha \in T^{\geq n}(V)$ satisfies $d \alpha=0$ and $[\alpha] \neq 0$.

Now define $\beta: T(U) \otimes(k \oplus M) \rightarrow T(X) \otimes(k \oplus N)$ by

$$
\beta(a+b)=a \otimes \alpha+\omega_{x}^{-1}\left(\omega_{U}(b) \otimes \alpha\right) \text { for } a \in T(U) \text { and } b \in T(U) \otimes M .
$$

We use the fact that $D \omega^{-1}=\mathrm{id}-\omega^{-1} d$ to show that $\beta$ commutes with the differentials: First, $D \beta(a+b)=d a \otimes \alpha+\omega_{U}(b) \otimes \alpha-\omega_{x}^{-1}\left(d \omega_{U}(b) \otimes \alpha\right)$. On the other hand, $\beta(d a+D b)=d a \otimes \alpha+\beta(D b)$; but $D b=D \omega_{U}^{-1} \omega_{U}(b)=$ $\omega_{U}(b)-\omega_{U}^{-1} d \omega_{U}(b)$, so $D \beta=\beta D$ is clear.

Choose a graded subspace $E \subset T(V)$ containing $d(T(V))$ such that $k\langle\alpha\rangle \oplus$ $E=T(V)$. Use this to define a lefi-T(U) module map $\Gamma: T(U) \otimes T(V) \rightarrow$ $T(U)$ so that $\Gamma(1 \otimes \alpha)=1$.

We can now define a left- $T(U)$ module retract

$$
r:(T(U) \otimes(k \oplus M), D) \rightarrow(T(U), d)
$$

by $r=\Gamma \circ \varphi \circ R \circ \beta$. It follows that $\operatorname{lMcat}(A) \leq m$, and this completes the proof of the theorem.

\section{REFERENCES}

[FH] Yves Felix and Stephen Halperin, Rational L.-S. category and its applications, Trans. Amer. Math. Soc. 273 (1982), 1-37.

[FHLT] Yves Felix, Stephen Halperin, Jean-Michel Lemaire, and Jean-Claude Thomas, Mod $p$ loop space homology, Invent. Math. 95 (1989), 247-262.

[H] Kathryn Hess, A proof of Ganea's conjecture for rational spaces, Topology 30 (1991), 205214.

[1] E. Idrissi, Quelques contre-exemples pour la L. S. catégorie d'une algèbre différentielle, Ann. Inst. Fourier (Grenoble) 41 (1991), 989-1003.

[HL] S. Halperin and J.-M. Lemaire, Notions of category in differential algebra, Lecture Notes in Math., vol. 1318, Springer-Verlag, Berlin and New York, 1988, pp. 138-154.

[J] Barry Jessup, Rational L.-S. category and a conjecture of Ganea, J. Pure Appl. Algebra 65 1990, 57-67.

[N] Bitjong Ndombol, Sur la catégorie de Lusternik-Schnirelmann des algèbres de cochaînes, Ann. Inst. Fourier (Grenoble) 41 (1991), 937-987.

Department of Mathematics, University of Ottawa, 585 King Edward Avenue, OtTawa, Ontario, Canada K1N 6N5

E-mail address: bjessup@acadvm1.uottawa.ca

Faculté des Sciences, Defartement de Mathématiques, Université de Yaounde, B.P. 812 YAOUNDE, CAMEROON 